\title{
Research on English Translation Skills in the Background of Functional Translation Theory
}

\author{
Yuehua Ji \\ English Department, School of Public Fundamentals, Jiangsu Vocational College of Medicine, \\ Yancheng 224006, China
}

Keywords: English translation; Theoretical perspective; Translation skills

\begin{abstract}
In the era of economic and technological development, global cultural exchanges and communication are also more frequent, and English as the world's first common language, the role of international exchange is obvious to all. Therefore, English translation is becoming increasingly important in our lives. Functional translation theory is a new perspective in translation theory research. The purpose of translation and the function of translation are the core work of functional translation. This paper mainly analyzes the daily translation skills of English from the perspective of functional translation theory.
\end{abstract}

\section{Introduction}

The main feature of English translation in the context of functional translation theory is strong purpose. The meaning of English translation is the conversion between two or more languages, which means that we can translate English into Chinese or translate German into English. In the translation, you need to pay attention to the following two aspects, first of all, the language form we issued, and then convert the original language form into the language content that needs to be translated through the intermediate media environment. Therefore, the following is an analysis of English translation skills from the perspective of functionalism.

\section{Functionalism and the concept of culture-specific items}

\subsection{Research on functionalism}

Functionalism originated in the 1970s with Katharina Reiss, Hans Vemeer, Christiane Nord and Justa Holz Manttari as the main representatives. Previously, most western translation theorists held the linguistic translation theory, namely the translation equivalence theory, which regarded translation as a process similar to cryptography, and advocated the original text as the center, focusing on the original text and the translation language. This saves the sign of the original text. The functional translation theorists believe that the equivalence theory has no way to do it in practice, and in many cases, it is not necessary. Vermeer proposed the translation function theory. He believed that the translation process was determined by the purpose of translation. The translator's reference system in the whole translation process should not be the original text and 
function required by the equivalent translation theory, but should be the translation. The communicative function expected in the context of the translated language culture. Subsequently, Nord proposed the concept of "function plus loyalty", stating that translation is a translated text that authorizes it to perform a certain function, and its connection with its original text is determined by the expected or required function of the translation. Due to language barriers and cultural differences among ethnic groups, an important factor to be concerned in the translation process is the audience. This theory emphasizes that translation is based on the behavior of a certain text, but the original text only provides the translator with a part of the multi-source information, allowing the translator to selectively translate, and there must be a certain relationship between the original and the translation. The quality and quantity of the contact is determined by the expected translation function. Nod provides the standard for which components in the original text in a specific context can be retained, and which components must be adjusted or even rewritten according to the context of the translation ${ }^{[1]}$.

\subsection{Research on culture-specific items}

Javier Franco Aixela, Spanish translator and translation theorist, defined culture-specific items in his book Culture-specific Items in Translation. It is difficult to convert the function and meaning of some items appearing in the original text to the translation because there is no corresponding item in the cultural system of the target language or have different text status with the items. The so-called different text status, that is, different values due to differences in ideology, idiom or frequency of use. Aixela explained that the purpose of his definition is to show that what is a culturally exclusive item does not depend solely on the project itself, but also on the function of the project in the text as understood by the translated language culture. That is to say, all ordinary readers or any translators in the culture of translation language feel that they are incomprehensible or unacceptable in terms of ideology or culture, that is, culturally exclusive items ${ }^{[2]}$.

\section{Principle of functional translation}

\subsection{Skopos rule}

In advancing learning tasks and work, it is necessary to set a goal. Only in this way can people continue to progress, and the process can be carried out more orderly, especially in English translation. The top-ranking rule for any translation is the scope rule, which means that a translation action is determined by its skopos, and is also extended to meet the needs of the key points of the translation effect. Since ancient times, the cultural connotations and forms of Chinese and Western cultures have been very different. Therefore, in order to achieve efficient communication between the two parties, this needs to be done through translation, and this form of translation must be recognized by both parties, so this requires translation. The cultural background and acceptability of the subject are considered comprehensively. Under normal circumstances, the differences in cultural background will directly reveal the differences in translation skills. For example, when conducting a normal conversation, you can try to choose a translation method with a literal meaning that is easy to understand, but you need to be cautious about the syntactic structure, and the words should be considered to ensure that the translated content is roughly consistent with the actual expression. At present, the global economic level is rising, and the exchanges between countries are becoming more frequent. Coupled with the continuous optimization and innovation of network communication technology, people understand that the world no longer needs to go abroad, only through Internet technology, in any Time and any place, as long as needed, can learn and understand foreign culture through a variety of media, so translation is becoming more and more important. 
Generally speaking, foreign film and television works can be spread to China and loved and promoted by our people. This is directly related to the level of English translation, but it is different from English translation in the form of diplomacy. It is only service. In the relevant field of translation, therefore, this further illustrates that functional translation is targeted in the process of translation to promote the deepening of translation content ${ }^{[5]}$.

\subsection{Fidelity rule}

Converting two different language forms to each other is not only a language exchange, but also a combination and collision between cultures. Therefore, the translation process is not as simple as imagined. It requires that the translators should have not only strong language translation level but also need to fully understand the content of culture between the objects of translation. Only in this way can the translation content be accurately displayed on the basis of ensuring the timeliness, and the mutual conversion between English and Chinese can be realized. If there are more differences between the content of the translation and the original text, or the general meaning of the translation does not match the original text, or some translators translate the occurrence of the character event in the translation process, etc., the translation is of no application value, and the translation loses its original meaning. Functional translation is based on the original text, and does not change the meaning of the original text, but also translates the content into a highly accurate demand language to the receiving party, but the accuracy mentioned here is not to translate verbatim and maintain every word of the translation in consistence with the original text. Instead, it should grasp the target of translation and remove the dross, so that the essence of the original text can be displayed in the translation.

\section{English Translation Skills under Functional Translation Theory}

\subsection{Free translation skills}

Free translation means that the translation strives to achieve flexible equivalence, and the target readers can get the same feelings as the original readers. Translation is to preserve the original form to the extent possible, but is not bound by the original form, and strives to preserve the original content, style, feelings and mood, including literal and implied meaning. The target language is naturally smooth, and the meaning and the style are the same.

In the case of many differences between the two languages, in order to achieve the above objectives, major changes can be made in wording and structure. Under the premise of functional equivalence, while eliminating language differences, there is no preservation of verbal features. For example, if the rhetorical form and national characteristics of the original text are not preserved, the words have the translation, the repetition, the change of the part of speech and the sentence pattern, the reverse of the word order, the disassembly of the sentence, the combination of the words, the positive and the opposite, and the translation. Omission, borrowing, and modification are all classified as free translation. Because the nature of these methods is common, there is no need to distinguish them finely.

\subsection{Literal translation skills}

Literal translation refers to keeping the original language form as much as possible, including words, sentence structure, metaphor, etc., while requiring the language to be fluent and easy to understand. Literal translation is guided by the source text structure, which can best preserve the cultural and national characteristics of the source language. The expression suggests that if a 
form-dependent translation achieves functional equivalence in the sensed and associative senses, there is no need to change the form ${ }^{[3]}$. Therefore, some Chinese cultural exclusive items can be translated literally. For example, (1) Many companies have adopted the management method of rewarding laziness and laziness to mobilize the enthusiasm of workers. A lot of companies have aroused the workers 'enthusiasm by adopting the way of awarding the diligent and punishing the lazy. The phrase "sports and lazy lazy" is translated literally, making the reader clear at a glance. (2) The mirror can't make the ugly ugly beautiful, nor can it make the social problems disappear. Smashing a mirror is no way to make an ugly person beautiful, nor is it a way to make social problems evaporate. (3) The receptionist used the method of "opening a lock with a key" to publicize the legal system and solve problems for the masses. The receiving personnel used the way of "opening different locks with different keys" in propagating the legal system and solving problems and alleviating sufferings for the people. The second and third sentences retain the original method of the original text, and the vivid image of the original text is unmistakable, achieving the language effect similar to the original text, making the language more vivid and vivid.

\subsection{Transliteration skills}

In translation, we all know that there is a certain difference between the target language and the original language. English translation is the same. If the translators pay attention to the importance of the source language, it is very easy to cause problems in translation, not the desired result of the target language. So that the reader cannot fully understand the meaning of the source text. On this basis, the person who translates the article needs to use the transliteration techniques to achieve the translation meaning of the target language. Let the readers better understand the meaning of the source language, and then express it in the expression of the target language.

For example, tangyuan (originally translated as: dumpling), man-tou (mantou), dumpling (jiaozi), and such words as wushu (martial arts), dazibao (big print), Yang (yang), Yin (yin), feng shui (feng shui) and other words have been found in the 1998 edition of the New Oxford Dictionary of English. In addition, the transliteration of Chinese food is also accepted by foreigners, for example, guobaorou, liurouduan.

\subsection{Adjustment skills}

To achieve a satisfactory functional equivalence translation, it is sometimes necessary to use the "method of adjustments" proposed by Nida. In the translation of cultural proprietary items, it is necessary to understand the principles of translation in order to understand the translation rules and improve the quality of translation. Fan Zhongying mentioned three translation principles in the practical translation course: (1) meaning transference, (2) acceptability, and (3) similarity. Some translations have played a guiding role. If any translation can't express the original meaning and make the target reader understand the meaning of the original text, then it will lose the meaning of translation, and it will not become a translation. The expression of thought must be carried out through language. The task of translation is to re-express the things expressed by the primitive carrier with the translated language. In most cases, the translated language is different from the original, the expression of the same thing, the word sentence and translation used by the primitive carrier. The language carrier is inconsistent. Therefore, you must not source when translating.

\subsection{Alienation and domestication skills}

In the history of Chinese translation, the dispute between alienation and domestication is seen in the large-scale debate between literal translation and free translation, represented by "faithfulness" 
and "expressiveness" in modern history. In recent years, domestic foreign language journals have published many articles on domestication and foreignization. These articles strive to apply the discussion of domestication and foreignization in Western literary criticism and comparative literary theory to translation theory and practice. The cultural perspective summarizes literal translation and free translation. These articles have broadened the research of domestication and foreignization from different angles. The dispute between domestication and foreignization has become a hot topic in translation theory. We must understand that the translation process is not only the activity of converting one language into another, but also a cultural exchange activity. With international globalization and trade between countries, cultural exchange activities are becoming more frequent. In the cultural exchanges between countries, domestication and foreignization play an increasingly important role in translation activities.

\section{Conclusion}

According to the above analysis, we can't do without an important theory in many of our current language translation work, that is, functional translation theory, especially in English translation, we should use translation skills derived from functional translation theory. First, understand the meaning or intention of the source language, and follow the principles of functional translation, and grasp the central idea of the source language according to their own understanding, and then translate into the target language according to the grammatical content of the target language, thus translate articles with fewer mistakes and let the recipients understand the meaning of the translation.

\section{References}

[1] Chai Guoxi; Wen Qiaozhi. Research on English Translation Skills Based on Chinese Tea Culture. Fujian Tea, Vol.10 (2017) No.15, p. 136-150.

[2] Chen Bowei. On the language characteristics and translation skills of news English. China Newspaper Industry, Vol.9 (2017) No.23, p. 11-15.

[3] He Peng. Exploring the Basic Strategy of Business English Translation Based on Application Function. Shandong Social Sciences. Vol.1 (2015) No.25, p. 33-35.

[4] Zhang Xiaoyan. Chinese Translation Skills of English Film and Television Works. Contemporary Television, Vol.11 (2015) No.17, p. 48-51.

[5] Zhao Xiulian. Exploration of Modern English Translation Skills - Comment on Business English Translation Course (Interpretation), China Education Journal, Vol.4 (2017) No.12, p. 26-29. 